\title{
ULTRASTRUCTURAL CHANGES IN HEPATOCYTES OF A POTENTIAL ANIMAL MODEL OF REYE'S SYNDROME
}

\author{
Devendra R. Deshmikh, Robert H. Gray, Merle Mason, Hunein F. Maassab, \\ and Joseph V. Baublis \\ Departments of Blological Chemistry, Environmental and Industrial Health, \\ Epidemiology, and Pediatrics, The University of Michigan, \\ Ann Arbor, Michigan 48109
}

\begin{abstract}
The onset of the childhood disorder, Reye's Syndrome (RS), is preceded by a viral infection, usually influenza. The syndrome is characterized by a series of clinical, metabolic, and morphologlcal changes which often progress to result in severe encephalopathy with Ilfe-threatening increases in intracranial pressure. Research on RS has been limited by the lack of an antmal model which can be induced to develop RS-like disorders following influenza infections. Unlike other laboratory species, ferrets are readily susceptible to influenza infections and exhiblt symptoms similar to those in children. Deshmukh, Kao, and Mason observed that young male ferrets fed a small amount of arginine deficient (AD) diet develop RS-like disorders after two to three hours, including hyperammonemia and an encephalopathy, that progress to coma and occasionally to death. Subsequent studies showed that infecting the arginine deficient ferrets with influenza $B$ results in a more severe and protracted encephalopathy with a much higher number of deaths. Signs of hepatic dysfunction, a major feature of RS, were also markedly increased in the infected-deficient ferrets. The ferrets used for the infection experiments were fed an arginine-restricted diet, containing $35 \%$ of the arginine content of a complete synthetic diet, for 6 days before infection. Two days following infection, they were fasted overnight for 16 hours, and then were provided for 2 hours with the synthetic $A D$ diet. An encephalopathy developed within 2 to 3 hours of presentation of the latter diet and rapidly progressed to a coma. Eight hours following the onset of encephalopathy, the comatose ferrets were sacrificed and tissue samples were taken for light and electron microscopy. Similar samples were taken from uninfected ferrets maintained during the same period, except for the period of fasting, on Purina Cat Chow. Microscopic examination of liver tissues revealed the following abnormalities in the infected-deficient animals as compared with those fed the chow diet (Fig. 1A): numerous microvesicular lipid droplets throughout the liver, glycogen depletion, pleomorphic mitochondria with the dense matrix granules present in reduced numbers, irregularly shaped peroxisomes with reduced electron density, and isolated regions of dilated endoplasmic reticulum (Fig. 1B-ID). These ultrastructural changes parallel the abnormalities reported in children with RS and, combined with other studies, suggest that further characterization of this unique animal model will be useful in future studies of the etiopathology of RS.

(Supported in part by NIH Grant AM-18647-05 and NIH Contract 1-A1-72551.)
\end{abstract}

Keywords: Reye's Syndrome, ferret, liver, mitochondria, peroxisomes, dilated rough endoplasmic reticulum, encephalopathy, influenza vims, animal model 


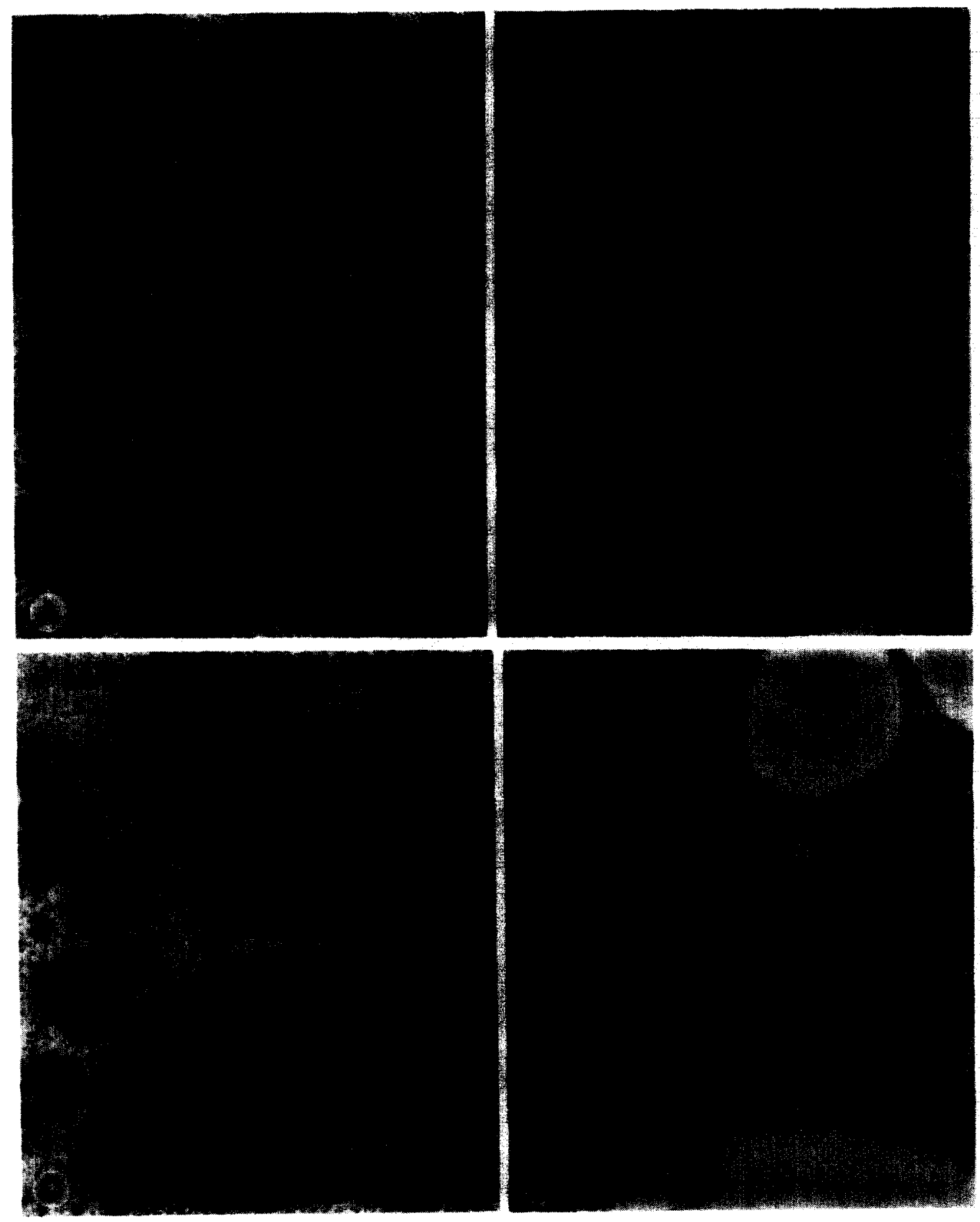

F1g, 1. Transmission electron micrographs of ferret hepatocyte, A. Control, ferrets maintained on Purina Cat Chow; B, C \&D. Fxperimental animals infected and fed modified diets (see text). Abbreviations: bile canaliculus (BC), dilated rough endoplasmic reticulum (D), glycogen (G), lysosome (L), mitochondria (M), mitochondria matrix granules (MG), nucleus $(N)$, peroxisomes (P), and rough endoplasmlc reticulum (RER). 